\title{
HTSC MULTILAYERS FOR DEVICE APPLICATIONS
}

\section{デバイス応用のための $\mathrm{H}$ i g h $-\mathrm{T}$ c 多層膜}

\author{
I enar i IGUCHI
}

Institute of Materials Science, University of Tsukuba

Tsukuba, Ibaraki, 305 JAPAN

TEL: 81-298-53-5128 FAX: 81-298-55-7440

(Received 10, October 1991 Accepted 28, November 1991)

\begin{abstract}
Studies on the thin-film multilayer structures of tunnel junction (S/I/S') and proximity tunnel junction (S/N/I/S') geometries are presented (S: HTSC, S':LTSC, N: normal material, I: insulator). The observed tunnel and Josephson characteristics are reported and discussed. A new tunneling model taking the special features of high $T_{C}$ superconductors into account is proposed to interpret the observed data. Some data on the proximity tunneling effect are also presented.
\end{abstract}

Keywords: High $T_{C}$ superconductor, Tunnel junction, Josephson effect, Proximity effect

\section{INTRODUCTION}

The fabrication and study of HTSC multilayer films are quite essential for the development of possible superconductive electronics based on high $T_{C}$ materials. The tunneling and Josephson effects are basic phenomena useful for device operation. The Josephson phenomena have been observed mostly using grain boundary junctions and $S / N / S$ (or $S^{\prime}$ ) junctions. The quasiparticle tunneling phenomena were rather complicated and not reproducible at an early stage of high $T_{C}$ superconductor research?due to the special features of high $T_{C}$ superconductors including the surface degradation, the extremely short coherence length and the gap anisotropy. The recent development of junction fabrication technique has, however, provided the reproducible tunnel junctions. ${ }^{2-5}$ The studies on the proximity-induced $S / N / S$ (or $S^{\prime}$ ) sandwich structures are now abundant. Quite recently, a long-range-proximity like phenomenon making use of an oxide material as $N$ layer has been reported. ${ }^{6}$ The essential nature of this phenomenon including the possibility of trivial or nontrivial effect has not been clarified yet.

In this presentation, the quasiparticle tunneling and Josephson tunneling effects observed in the multilayer planar YBCO/I/Pb junctions are reported. The observed phenomena are interpreted by a newly proposed tunneling model by taking the special features of high $T_{C}$ materials such as the extremely short coherence length, the gap anisotropy, the surface degradation, the surface inhomogeneities into account. 7,8 With regards to a long range proximity effect, some data on the tunnel characteristics of $\mathrm{YBCO} / \mathrm{N} / \mathrm{I} / \mathrm{Pb}$ proximity tunneling junctions ( $\mathrm{N}$ : normal metal or oxide materia 1 ) are presented and discussed.

\section{EXPERIMENTAL}

The fabrication of sandwich type tunnel junctions was performed utilizing an electron- 
beam and thermal heater coevaporation chamber equipped with an in situ metal mask exchange system. Both the $\mathrm{YBCO} / \mathrm{I} / \mathrm{Pb}$ junctions with artificial ( $\mathrm{MgO}, \mathrm{Y}_{2} \mathrm{O}_{3}$ of a few $\mathrm{nm}$ thick) and native barriers were fabricated, whose details are described in Refs. 3 and 4 . The c-axis and $\mathrm{a}(\mathrm{b})$-axes oriented $\mathrm{YBCO}$ films were grown on $\mathrm{MgO}, \mathrm{SrTiO}_{3}$, and $\mathrm{LaSrGaO}_{4}(100)$ substrates respectively. For the latter, it was a a(b)axes oriented film with some inclusion of caxis oriented regions ( $5-20 \%)$ grown under specific condition. The native barrier was formed either by in-situ annealing at 400-500 ${ }^{\circ} \mathrm{C}$ for $\mathrm{hr}$ in the chamber or by exposing a YBCO film to the ambient atmosphere. Josephson current was observable for junctions with an artificial barrier of less than $2 \mathrm{~nm}$ thick and for those with a native barrier grown in situ.

\section{TUNNEL AND JOSEPHSON CHARACTERISTICS}

Figure 1 shows an example of observed tunnel characteristic ( $d V / d I$ vs $V$ ) whose gap edge behavior appeared clearly. In addition to this structure, the zero bias anomaly (conductance enhancement) and the sharp gap structure around zero bias are visible. The absence of BCS gap bump and the presence of $\mathrm{Pb}$ gap structure at zero bias are quite unusual in the light of the conventional tunneling theory.

The zero bias anomaly was typical for the junctions with smaller tunnel resistance $\left(10^{-4}\right.$ $-10^{-2} \Omega \mathrm{cm}^{2}$ ) and probably reflects the contribution from some additional effect. The zero bias anomaly has been also reported by many other authors, which was found to be strong for YBCO junctions and weak or almost absent for BiSrCaCuO junctions. The linearly rising conductance above the gap edge was also consistent with the numerous data reported previous1y. The tunnel characteristics observed for the junctions with the c-axis and $a(b)$-axes oriented YBCO films were qualitatively similar except that the $\mathrm{YBCO}$ gap-edge structure was more pronounced for the latter.

Figure 2 shows the Josephson I-V curves

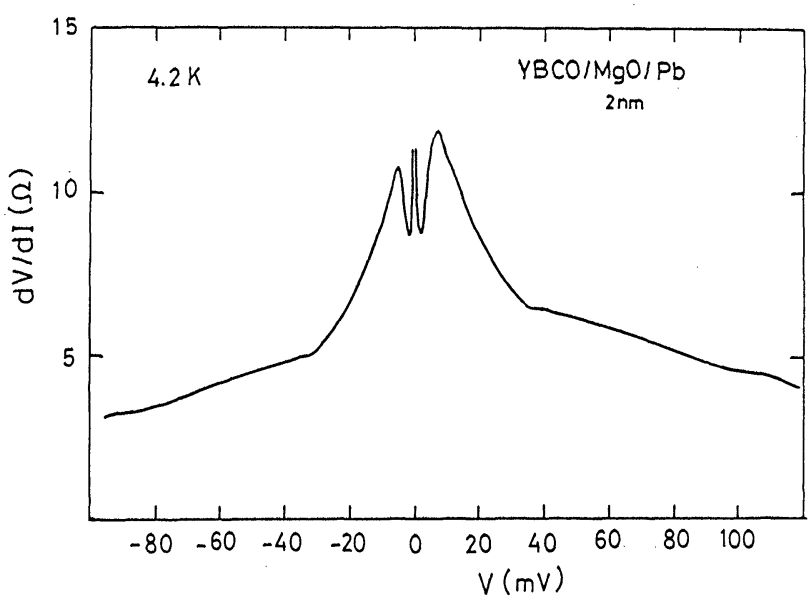

Fig. 1. Tunnel characteristic of a $\mathrm{YBCO} / \mathrm{MgO} / \mathrm{Pb}$ junction ( $\mathrm{MgO}: 2 \mathrm{~nm})$ at $4.2 \mathrm{~K}$. c-axis YBCO

$4.2 \mathrm{~K}$

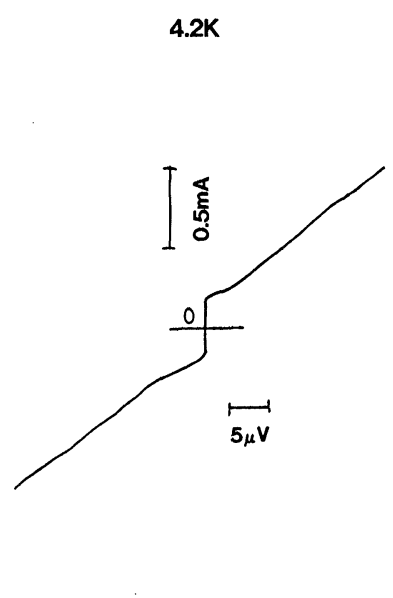

(a)

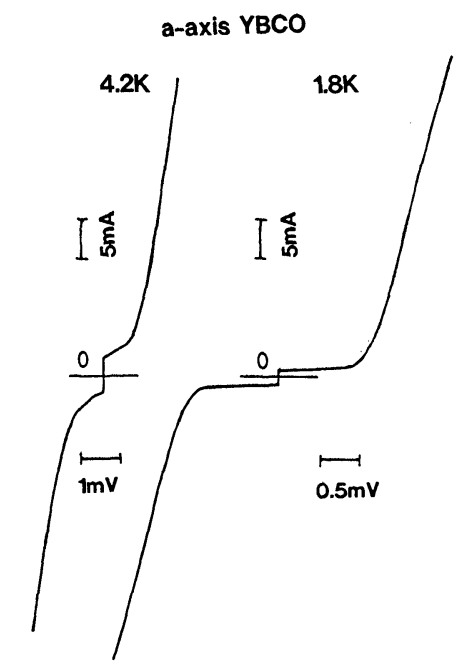

(b)
Fig.2. Josephson I-V characteristics for YBCO/ native barrier/Pb tunnel junctions with the $c$-axis (a) and the $a(b)$-axes (b) oriented YBCO films.

observed for the junctions with c-axis and a(b) -axes oriented YBCO films. The maximum Josephson current was typical1y 0.05-0.2mA for the c-axis oriented $f i 1 \mathrm{~m}$ and $0.5-3 \mathrm{~mA}$ for the $a(b)$-axes oriented $f i 7 m$. There apparently exists the qualitative difference between (a) and (b) in which weak link behavior is obser- 
vable for the curve (a) whereas highly nonlinear behavior (almost switching behavior toward the $\mathrm{Pb}$ gap curve) for the curve (b). The $I-V$ curve (a) in the finite voltage was, however, found to follow the $\mathrm{Pb}$ gap curve in larger voltage scale of $\mathrm{mV}$ order. The Josephson nature of zero voltage current in Fig.2 was demonstrated by observing Shapiro steps under microwave irradiation of $9 \mathrm{GHz}$ and Fraunhofer diffraction like pattern under an applied magnetic field. 4,9

The temperature dependence of Josephson maximum current was found to be very anomalous since it decreased as the bath temperature went down below about 5K. Figure 3 depicts such result for many $\mathrm{YBCO} / \mathrm{I} / \mathrm{Pb}$ junctions. 10 We point out that the normal tunnel resistance of junctions did not change appreciably below $7.2 \mathrm{~K}$ so that the conventional AmbegaokarBaratoff theory cannot explain the observed results. 11 The study of Fraunhofer-1ike pattern at different temperatures led a new fact that Josephson maximum current became insentive to magnetic field at lower temperatures. Hence the observed phenomena may be

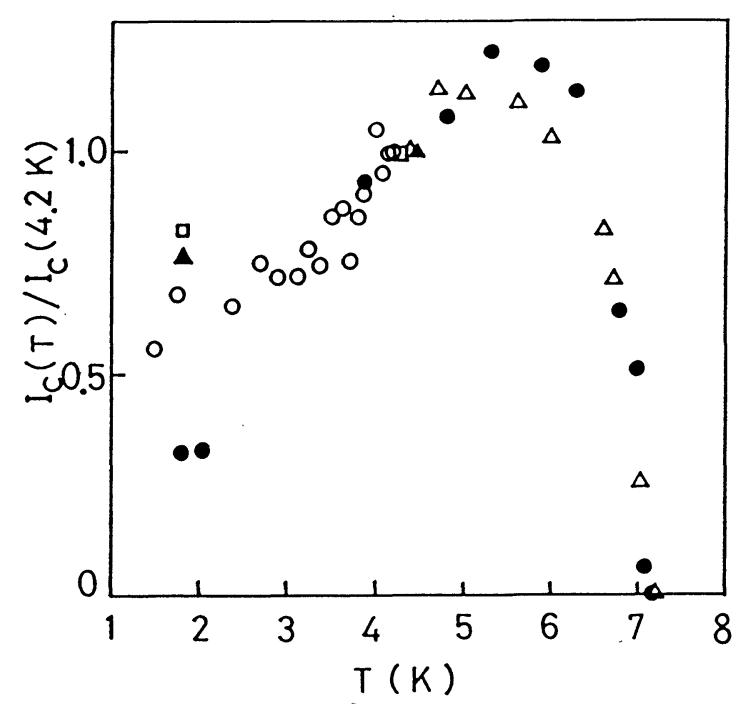

Fig.3. Temperature dependence of Josephson maximum current for a $\mathrm{YBCO} / \mathrm{I} / \mathrm{Pb}$ junction. related to some flux frozen effect at low temperatures. 12

\section{TUNNELING MODEL}

In order to interpret the observed results, we first pay attention to possible real situation in the tunneling process expected for high $T_{C}$ material. Figure 4 sketches a crosssectional view of a real tunnel junction with an artificial barrier when the surface inhomogeneity in an atomic scale is present. The YBCO film was assumed to be c-axis oriented. In this case, both the tunneling into the c-axis and the ab-plane may be expected, in which each contribution may strongly depend on the surface roughness.

Figure 5 shows a schematic semiconductor tunnel diagram for the real tunneling situation corresponding to Fig. 4 which takes the gap anisotropy, the surface degradation and the surface inhomogeneity. In the tunneling into the ab-plane (process (a)), the conventional quasiparticle tunneling is expected. In the tunneling into the c-axis (process (b)), the electrons are first injected into a normal layer of YBCO which would be produced by surface degradation effect due to oxygen deficiency, then they are reflected by the pair potential of the c-axis $\Delta_{c}$. Hence, for the

\section{$\mathrm{Pb}$}

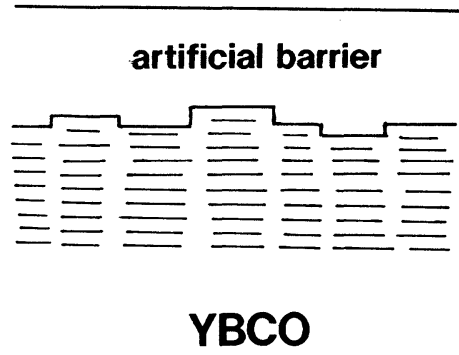

Fig.4. A schematic of the cross-sectional view of a real $\mathrm{YBCO} / \mathrm{I} / \mathrm{Pb}$ tunnel junction. 
(a)

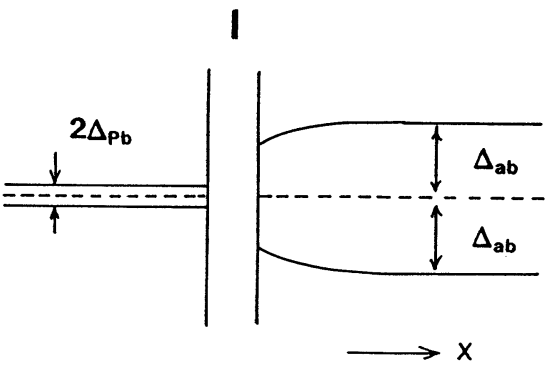

(ab plane)

(b)

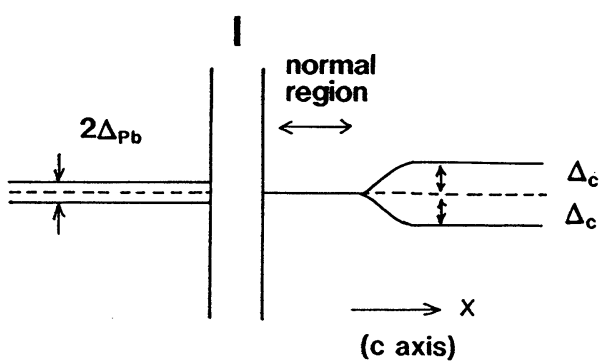

Fig.5. Tunneling scheme expected for a YBCO/I/ $\mathrm{Pb}$ tunnel junction with an artificial barrier when the surface inhomogeneity is present (see Fig.4). $\Delta_{\mathrm{ab}}, \Delta_{\mathrm{c}}$ and $\Delta_{\mathrm{Pb}}$ are the gap parameters of YBCO in the ab-plane, YBCO along the $c$-axis and $\mathrm{Pb}$ respectively. The surface of a $\mathrm{c}$-axis oriented $f i 7 m$ was assumed to be normal due to surface degradation.

Tatter, $\mathrm{Pb} / \mathrm{I} /$ normal tunneling and Andreev reflection 13,14 are possible to occur. The $\mathrm{S} / \mathrm{N}$ barrier strength paramter inside $\mathrm{YBCO}$ material is considered to be quite small because the $S / N$ barrier is naturally formed by oxygen deficiency (there is no change of crystal structure at the boundary), hence the probability of Andreev reflection is expected to be large. The observed tunnel conductance $G_{o b s}$ becomes the sum of two processes in Fig.5,i.e. $G_{\text {obs }}=G_{a b}+G_{c}$ where $G_{c}=\left(R_{j}+R_{A}\right)^{-1}\left(R_{J}\right.$ : $\mathrm{Pb} / \mathrm{I} / \mathrm{N}(\mathrm{YBCO})$ junction resistance, $R_{A}$ : Andreev resistance).

Then the expected conductance $G_{o b s}$ is depicted in Fig.6, whose behavior quite resembles Fig.1. In case of the junctions with a native barrier, because of strong degradation of a YBCO film from the surface, an order parameter varying in a long range scale toward the thick-

\section{$\mathrm{T}<\mathrm{T}_{\mathrm{c}}(\mathrm{Pb})$}

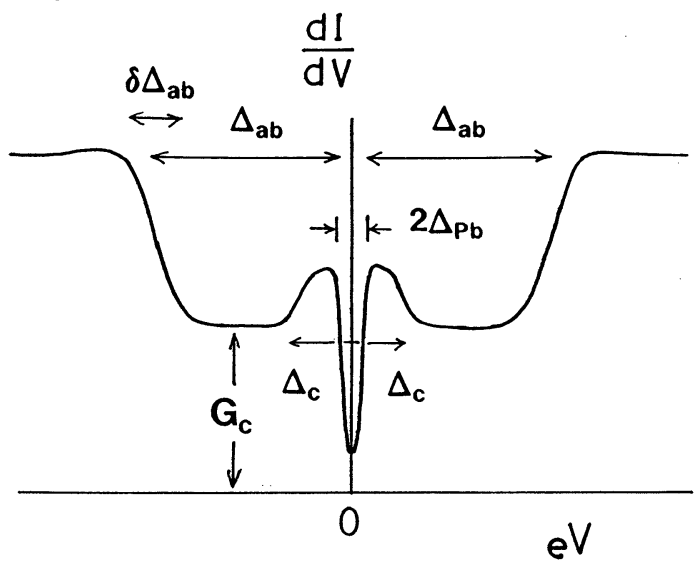

Fig.6. Tunnel conductance curve expected from the tunneling scheme of Fig.5. $\delta \Delta_{a b}$ is the gap broadening due to the local smearing near the surface. $G_{C}$ is the conductance due to the process (b) in Fig.5. For $T>T_{C}(P b)$, the $\mathrm{Pb}$ peak disappears and the conductance enhancement around zero bias becomes clear.

ness direction across a film is expected, which yields a V-type shape behavior for the tunnel conductance as shown in Fig.7. A very weak zero bias anomaly is expected due to the weak order parameter along the c-axis. The result in Fig. 7 agrees with the recent measurements on the junctions with native barriers?, 7

Our new tunneling model is successful to interpret the observed finite conductance inside the gap region, the $\mathrm{Pb}$ gap structure around zero bias and the conductance enhancement around zero bias. The detailed shape of tunnel conductance curve depends on the relative contributions between the process (a) and (b). If a YBCO film is ideally c-axis oriented and and its surface is flat in an atomic scale, the $\Delta_{a b}$ structure will not appear.

According to the model given above, Josephson current will not flow along the c-axis direction. Hence the observed Josephson current along the c-axis direction in Fig. 2 is due to the surface roughness of a YBCO $\mathrm{fi} 1 \mathrm{~m}$ which allowed the Josephson tunneling into 
the ab-plane. The behavior in the finite voltage region is also interpreted by the above model. Figure 8 shows an equivalent circuit for the junction having the inhomogeneous YBCO film surface. Since $G_{a b}<<G_{c}$ generally, $G_{o b s} \simeq G_{C}$ in the smal1 voltage region

\section{$T>T_{c}(P b)$}

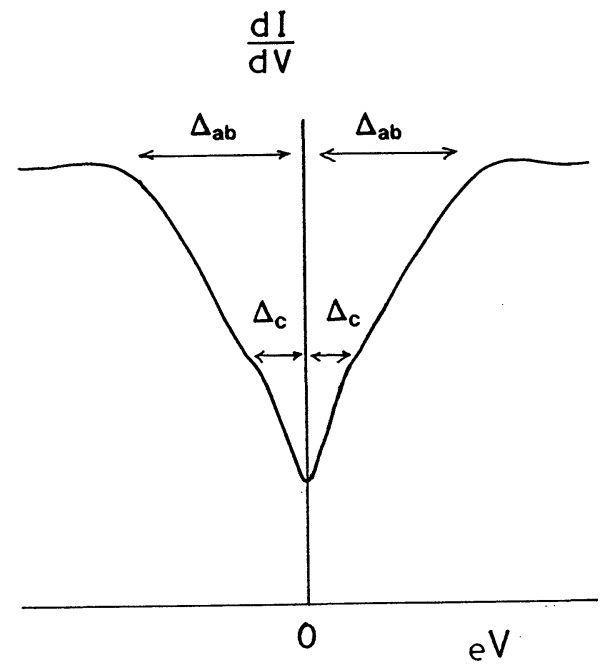

Fig.7. Tunnel conductance curve expected for a $\mathrm{YBCO} /$ native barrier/Pb tunnel junction. Due to the long range spatial variation of the order parameter at least for a few unit cells from the film surface and the short coherence length of a few $\mathrm{nm}$, a V-type conductance curve is obtained.

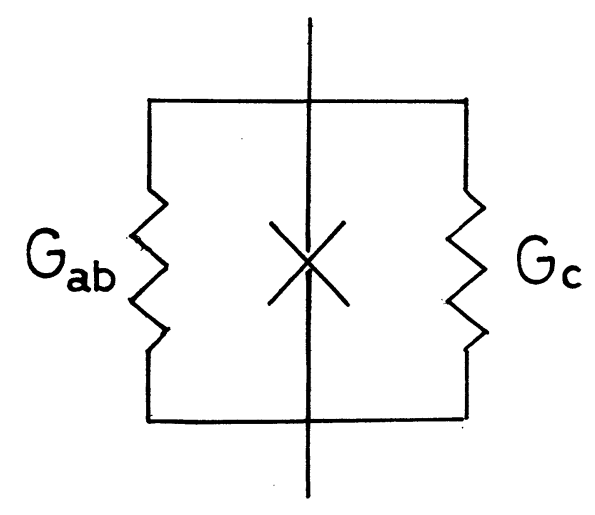

Fig. 8. Equivalent circuit for a Josephson tunnel junction when the surface inhomogeneity exists in a high $T_{C}$ superconducting $f i l m$.
$\left(\mathrm{V} \leq \Delta_{\mathrm{Pb}}\right)$. Then the $\mathrm{I}-\mathrm{V}$ curves are expected to follow the $\mathrm{Pb}$ gap curve, in consistent with the data in Fig.2. The strong nonlinearity in Fig.2(b) appeared because the magnitude of maximum Josephson current corresponded to that of a current rising portion of $\mathrm{Pb}$ gap structure.

\section{PROXIMITY TUNNELING RESULTS}

Next, in order to investigate the nature of YBCO gap parameter, the results on the proximity-induced effect using the YBCO/N/I/ $\mathrm{Pb}$ type multilayer junctions are presented. The measurements are important in the light of the recent stimulating reports on longrange-proximity like effect and also possible application to device technology.

As a normal layer $N, A T$ and $L a B a C u O ~ f i l m s$ were used. The multilayer structures were fabricated by an in situ deposition utilizing a metal mask system, the method similar to tunnel junctions. In case that an $A 1 \mathrm{film}$ was used, the tunnel barrier was formed by the rf plasma oxidation of the Al film for a few tens of min. In case that a LaBaCuO film was used, direct deposition of $\mathrm{Pb}$ on $\mathrm{LaBaCuO}$ was held. The native barrier was presumably formed by the reaction of materials.

The $d V / d I$ vs $V$ curves for $\mathrm{YBCO} / \mathrm{Al} / \mathrm{A} 10_{\mathrm{X}} / \mathrm{Pb}$ junctions at different $A 1$ thickness $\left(d_{A T}\right)$ showed that the $\mathrm{Pb}$ peak around zero bias was reduced and broadened as $\mathrm{d}_{\mathrm{A}}$ was increased and diminished away for $d_{A}>15 \mathrm{~nm}$. This happened probably because the Cooper pairs diffused three dimensionally in an Al film, thereby smoothing out the original inhomogeneities in a YBCO film.

The result for a $\mathrm{YBCO} / \mathrm{LaBaCuO} / \mathrm{Pb}$ junction (LaBaCu0: 50nm) are shown in Fig.9. Note that a LaBaCuO film was semiconductive although its composition differed from $\mathrm{La}_{1} \cdot 5^{\mathrm{Ba}}{ }_{1} \cdot 5^{\mathrm{Cu}_{3} \mathrm{O}_{\mathrm{y}}} 6$ In addition to the induced gap structure ( $\Delta \sim$ $10 \mathrm{meV}$ ) by the YBCO proximity effect, a Pb gap peak was still visible even after the tunnel 


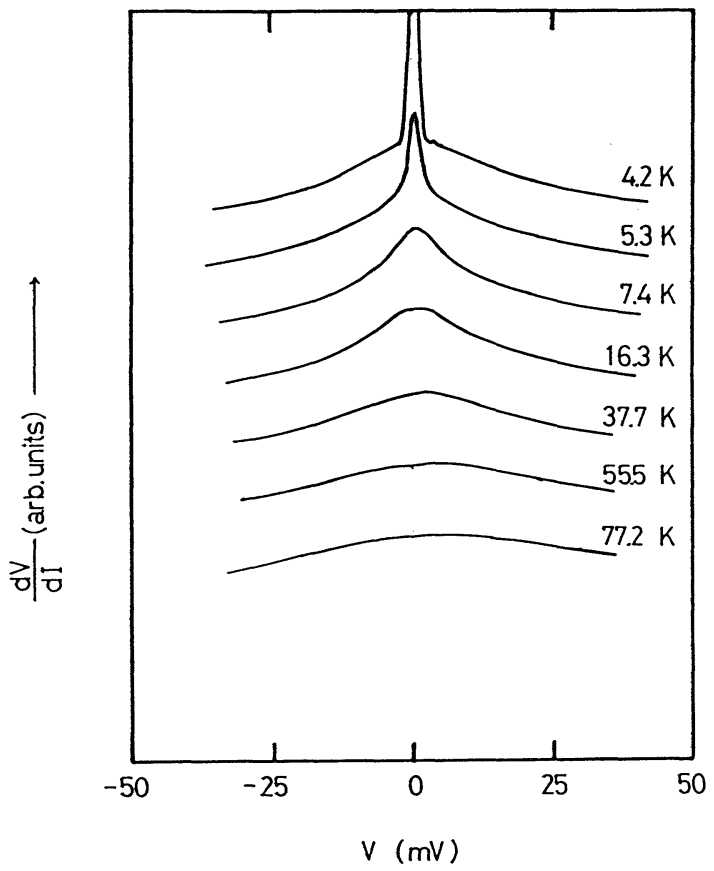

Fig.9. Tunnel characteristics (dV/dI vs $V$ ) for a $Y B C O / L a B a C u 0 / P b$ junction ( $\mathrm{LaBaCuO}: 50 \mathrm{~nm}$ ) at different temperatures.

current path of $50 \mathrm{~nm}$, suggesting that the local information about the inhomogeneities in a YBCO film was not smoothed out through a $\mathrm{LaBaCuO}$ film. In other words, the local information of YBCO pair wave function was transmitted to a tunnel detection area. The result suggests the formation of narrow superconducting path in a LaBaCuO film, being consistent with the idea of $S / S^{\prime} / S$ structure for the long-range proximity $S / N / S$ junctions.

\section{CONCLUDING REMARKS}

Final1y, we briefly discuss about the possible application of HTSC multilayer structures to electronic devices based on the observed results on tunnel junctions and proximity tunneling junctions, and the newly proposed model. For the fabrication of S/I/S junctions (S:HTSC), the formation of normal regions on the film surface especially for YBCO superconductors provides a troublesome problem. To get rid of this problem, either the growth of a film with purely $a(b)$-axes oriented having an atomically flat surface or the fabrication of high quality S/N/I/S tunnel junctions is necessary. With regards to the long range proximity like effect, the induced gap in an oxide material may be local, whose picture is consistent with the idea of $S / S^{\prime} / S$ Tike junction for the $\mathrm{HoBaCuO} / \mathrm{LaBaCuO} / \mathrm{HoBaCuO}$ structure. 6 The essential nature of this structure should be, however, clarified before the device application is considered.

\section{ACKNOWLEDGEMENTS}

The author would like to thank Dr. Z. Wen, Messrs. T. Kusumori and H. Ohtake and Miss M. Nasu for experimental assistance.

\section{REFERENCES}

${ }^{1}$ J. R. Kirtley, Int. J. Mod. Phys. B4, 201 (1990).

${ }^{2}$ M. Gurvitch et a1., Phys. Rev. Lett. 63, 1008 (1989).

${ }^{3}$ I. Iguchi et a1., Jpn. J. App 1. Phys. 29 L614 (1990).

${ }^{4} I$. Iguchi and $Z$. Wen, IEEE Trans, on MAG. 27, 3102 (1991).

${ }^{5}$ B. A. Aminov et al., to be published.

${ }^{6}$ Y. Tarutani et a1., Ext. Abstr. 3rd FED Workshop on HiTcSc-ED (1991), p. 190.

${ }^{7}$ I. Iguchi and $Z$. Wen, Physica C178, 1 (1991).

${ }^{8}$ I. Iguchi, Z. Wen and M. Nasu, Ext.Abstr. 3rd FED Workshop on HiTcSc-ED (1991), p. 166.

${ }^{9}$ Z. Wen, I. Iguchi and K. Nakamura, J. App 1. Phys. 69, 7363 (1991).

${ }^{10} \mathrm{Z}$. Wen and I. Iguchi, Jpn. J. App 1. Phys. 30, L188 (1991).

${ }^{11} \mathrm{~V}$. Ambegaokar and A. Baratoff, Phys. Rev. Lett. 10, 486 (1963); ibid. 11, 104 (1963).

${ }^{12}$ A. P. Malozemoff, private communication.

${ }^{13}$ A. F. Andreev, Sov. Phys. JETP 19, 1228 (1964).

${ }^{14} \mathrm{G}$. E. Blonder, M. Tinkham and T. M. Klapwijk, Phys. Rev. B25, 4515 (1982). 


\title{
The 2nd Tokai University International Workshop \\ on \\ SUPERCONDUCTIVITY \\ (TUIWS)
}

\author{
October 9-12, 1991
}

Tokai University Pacific Center

2241 Kapiolani Blvd. Honolulu, Hawaii

(Phone: 808-973-4100 Fax: 808-973-4107)

\section{CONFERENCE ORGANIZERS}

\section{Organizing Committee (Tokai University) \\ Chair; Tatsuro MATSUMAE \\ (President of Tokai University) \\ Sonosuke KATORI \\ Shoji YOSHIDA \\ Kimikazu MATSUYAMA \\ Norio MATSUMAE \\ Yoshio MIHARA \\ Hajime YANO \\ Takashi MATSUMOTO \\ Toru HIRAYAMA \\ Mamoru KAWAKAMI}

\section{Conference Organizers}

$\begin{array}{ll}\text { Chair; } & \text { Sadao NAKAJIMA } \\ \text { Co-Chair; } & \text { Shoji TANAKA } \\ \text { Co-Chair; } & \text { Kyoji TACHIKAWA } \\ & \text { Mamoru KAWAKAMI } \\ & \text { Hideomi KOINUMA }\end{array}$

\section{Program Committee}

Chair; Tadashi SHIRAISHI

Hideomi KOINUMA

Kazumasa TOGANO

Naoto NAGAOSA

Yoshitake NISHI

Yuh SHIOHARA

Secretariat

Chair; Tadashi SHIRAISHI

Ichiro NAKADA

Shusuke YOMO

Yasunari KURIHARA

Tetsunobu TAKAYAMA

Minoru HAYAMA

Masaaki IGARASHI 